\title{
Impact of the Absolute Difference in Diastolic Blood Pressure Between Arms in Patients With Coronary Artery Disease
}

\author{
Yuka Hitaka $^{\mathrm{a}}$, Shin-ichiro Miura, ${ }^{\mathrm{a}, \mathrm{b}, \mathrm{c}}$, Rie Koyoshi ${ }^{\mathrm{a}}$, Yuhei Shiga ${ }^{\mathrm{a}}$, Yuiko Miyase ${ }^{\mathrm{a}}$, Kenji Norimatsu ${ }^{\mathrm{a}}$,

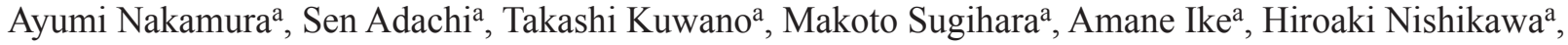 \\ Keijiro Saku ${ }^{\mathrm{a}, \mathrm{b}}$
}

\begin{abstract}
Background: We investigated the relationship between the severity and presence of coronary artery disease (CAD) and a difference in systolic and diastolic blood pressure (SBP and DBP) between arms or between lower limbs.

Methods: We enrolled 277 patients who underwent coronary angiography. We calculated the absolute (|right BP (rt. BP) - left BP (lt. BP)|) and relative (rt. BP - 1t. BP) differences in SBP or DBP between arms or between lower limbs, and assessed the severity of CAD in terms of the Gensini score.

Results: The absolute difference in DBP between arms in the CAD group was significantly lower than that in the non-CAD group, whereas the absolute difference in DBP between lower limbs in the CAD group was significantly higher. There were no differences in the absolute or relative difference in SBP between arms or lower limbs between the groups. The absolute difference in DBP between arms decreased as the Gensini score increased. In a logistic regression analysis, the presence of CAD was independently associated with the absolute difference in DBP between arms, in addition to male, family history, dyslipidemia, diabetes mellitus and hypertension.
\end{abstract}

Conclusion: The absolute difference in DBP between arms in addition to traditional factors may be a critical risk factor for the presence of CAD.

Keywords: Diastolic blood pressure; Coronary artery disease; Difference in blood pressure between arms; Traditional risk factors

Manuscript accepted for publication September 08, 2015

aDepartment of Cardiology, Fukuoka University School of Medicine, Fukuoka 814-0180, Japan

bDepartment of Molecular Cardiovascular Therapeutics, Fukuoka University School of Medicine, Fukuoka 814-0180, Japan

${ }^{\mathrm{c} C}$ Corresponding Author: Shin-ichiro Miura, Department of Cardiology, Fukuoka University School of Medicine, 7-45-1 Nanakuma, Jonan-ku, Fukuoka 814-0180, Japan.Email: miuras@cis.fukuoka-u.ac.jp

doi: http://dx.doi.org/10.14740/jocmr2330w

\section{Introduction}

According to the Japanese Society of Hypertension Guidelines for the Management of Hypertension 2014, bilateral brachial blood pressure (BP) should be measured at the initial clinic visit [1]. Generally, BP in the right arm is a few $\mathrm{mm} \mathrm{Hg}$ higher than that in the left arm [2]. However, a difference in systolic BP (SBP) of more than $10 \mathrm{~mm} \mathrm{Hg}$ between arms has been shown to be strongly associated with the presence of cardiovascular disease (CVD) and increased CV mortality [3-6]. We recently found that a relative difference in SBP between arms by synchronal measurement may be associated with the presence of coronary artery disease (CAD) [7]. Thus, a difference in BP between arms could be a useful indicator of the risk of CVD and mortality.

Since the difference in BP between arms could be a useful indicator of the risk of CVD, we hypothesized that a difference in BP could, along with traditional CV risk factors, predict the severity and presence of CAD. Therefore, we investigated whether the differences in SBP and DBP between arms and lower limbs were associated with the severity and presence of CAD.

\section{Methods}

\section{Subjects}

We enrolled 277 consecutive patients who were clinically suspected of having $\mathrm{CAD}$, and who had at least one cardiac risk factor or an abnormality in their electrocardiogram, such as ST depression, negative $\mathrm{T}$ wave, or left bundle branch block. All patients underwent coronary computed tomography angiography (CTA) or invasive coronary angiography (CAG) and an assessment of brachial-ankle pulse wave velocity (baPWV). We excluded patients with unstable angina or myocardial infarction within the previous 4 weeks, chronic renal disease with hemodialysis or peripheral artery disease. We divided the patients into two groups, a non-CAD group $(\mathrm{n}=86)$ and a CAD group $(n=191)$, and defined the severity of coronary atherosclerosis according to the Gensini score. Stable angina (CAD group) was defined as no changes in the frequency, duration, or intensity of symptoms for 4 weeks and as lumen diameter stenosis $>50 \%$ by CAG or CTA in at least one major 
Table 1. Patient Characteristics in All Patients, Non-CAD and CAD Groups

\begin{tabular}{|c|c|c|c|}
\hline & All $(n=277)$ & Non-CAD $(n=86)$ & CAD $(n=191)$ \\
\hline Age, years & $66.5 \pm 9.8$ & $65.2 \pm 10.8$ & $67.1 \pm 9.3$ \\
\hline Male, n (\%) & $193(70)$ & $47(55)$ & $146(76)^{*}$ \\
\hline BMI, $\mathrm{kg} / \mathrm{m}^{2}$ & $23.7 \pm 3.3$ & $23.3 \pm 3.3$ & $23.9 \pm 3.3$ \\
\hline Family history, n (\%) & $63(23)$ & $13(15)$ & $50(26)^{*}$ \\
\hline Smoking, n (\%) & $146(53)$ & $37(43)$ & $109(57)^{*}$ \\
\hline HTN, n (\%) & $213(77)$ & $53(62)$ & $160(84)^{*}$ \\
\hline DL, n (\%) & $232(84)$ & $58(67)$ & $174(91)^{*}$ \\
\hline $\mathrm{HDL}-\mathrm{C}, \mathrm{mg} / \mathrm{dL}$ & $52 \pm 13$ & $58 \pm 13$ & $50 \pm 12 *$ \\
\hline LDL-C, mg/dL & $103 \pm 30$ & $115 \pm 29$ & $97 \pm 29^{*}$ \\
\hline $\mathrm{TG}, \mathrm{mg} / \mathrm{dL}$ & $132 \pm 74$ & $124 \pm 61$ & $136 \pm 79$ \\
\hline DM, n (\%) & $107(39)$ & $17(20)$ & $90(47)^{*}$ \\
\hline $\mathrm{HbA} 1 \mathrm{c}, \%$ & $6.1 \pm 1.1$ & $5.8 \pm 0.8$ & $6.2 \pm 1.2 *$ \\
\hline HU, n (\%) & $50(18)$ & $15(17)$ & $35(18)$ \\
\hline $\mathrm{UA}, \mathrm{mg} / \mathrm{dL}$ & $5.4 \pm 1.3$ & $5.3 \pm 1.2$ & $5.5 \pm 1.3$ \\
\hline $\mathrm{eGFR}, \mathrm{mL} / \mathrm{min} / 1.73 \mathrm{~cm}^{2}$ & $64 \pm 16$ & $66 \pm 14$ & $63 \pm 16$ \\
\hline baPWV (mean), cm/s & $1,734 \pm 368$ & $1,694 \pm 315$ & $1,753 \pm 389$ \\
\hline \multicolumn{4}{|l|}{ Medication, n (\%) } \\
\hline ARB/ACE-I & 177 (64) & $14(16)$ & $47(25)^{*}$ \\
\hline $\mathrm{CCB}$ & $134(48)$ & $31(36)$ & $103(54)^{*}$ \\
\hline$\alpha$-blocker & $3(1)$ & 0 & $3(2)$ \\
\hline$\beta$-blocker & $43(16)$ & $8(9)$ & $35(18)^{*}$ \\
\hline Diuretics & $40(14)$ & $10(12)$ & $30(16)$ \\
\hline Statin & $192(69)$ & $32(37)$ & $160(84)^{*}$ \\
\hline Ezemitibe & $22(8)$ & $3(3)$ & $19(10)$ \\
\hline Insulin & $15(5)$ & $2(2)$ & $13(7)$ \\
\hline Sulfonyurea & $34(12)$ & -11 & $25(13)$ \\
\hline Pioglitazone & $6(2)$ & $1(1)$ & $5(3)$ \\
\hline
\end{tabular}

BMI: body mass index; HTN: hypertension; DL: dyslipidemia; HDL-C: high-density lipoprotein cholesterol; LDLC: low-density lipoprotein cholesterol; TG: triglyceride; DM: diabetes mellitus; HbA1c: hemoglobin A1c; HU: hyperurecemia; UA: uric acid; eGFR: estimated glomerular filtration rate; baPWV: brachial-ankle pulse wave velocity; ARB/ACE-I: angiotensin II receptor blocker/angiotensin converting enzyme inhibitor; CCB: calcium channel blocker. * $\mathrm{P}<0.05$ vs. non-CAD group.

coronary artery. The protocol in this study was approved by the Ethics Committee of Fukuoka University Hospital. We retrospectively collected and analyzed all data using the database of Fukuoka University Hospital.

\section{Assessment of CV risk factors}

Data on weight, height, serum levels of triglycerides (TG), high-density lipoprotein cholesterol (HDL-C), and low-density lipoprotein cholesterol (LDL-C), estimated glomerular filtration rate (eGFR), uric acid (UA) and hemoglobin A1c (HbA1c) were collected. The patient characteristics, including the history of hypertension (HTN), dyslipidemia (DL), diabetes mellitus (DM), smoking status (current versus non-smok- ers), family history (myocardial infarction, angina pectoris, or sudden death), and medication use were obtained from medical records. Patients who had a current SBP/DBP $\geq 140 / 90$ $\mathrm{mm} \mathrm{Hg}$ or who were receiving antihypertensive therapy were considered to have HTN. Patients with LDL-C $\geq 140 \mathrm{mg} / \mathrm{dL}$ and/or TG $\geq 150 \mathrm{mg} / \mathrm{dL}$ or HDL-C $\leq 40 \mathrm{mg} / \mathrm{dL}$, or who were receiving lipid-lowering therapy were defined as DL. DM was defined using the Japanese Diabetes Society criteria. Body mass index (BMI) was calculated as weight $(\mathrm{kg}) /$ height $^{2}\left(\mathrm{~m}^{2}\right)$.

\section{Measurement of baPWV}

BaPWV was measured with subjects in the supine position using a volume-plethysmographic device (PWV/ABI, OMRON 
Table 2. Rt. and Lt. BP, Absolute and Relative BP Differences Between Arms and Lower Limbs in All Patients, Non-CAD and CAD Groups

\begin{tabular}{|c|c|c|c|}
\hline & All $(n=277)$ & Non-CAD $(n=86)$ & $\operatorname{CAD}(n=191)$ \\
\hline Rt. SBP arm, mm Hg & $130.4 \pm 18.0$ & $132.8 \pm 19.3$ & $129.3 \pm 17.4$ \\
\hline Lt. SBP arm, mm Hg & $129.9 \pm 18.0$ & $132.5 \pm 18.7$ & $128.7 \pm 17.6$ \\
\hline M. SBP arm, mm Hg & $130.1 \pm 17.8$ & $132.6 \pm 18.7$ & $129.0 \pm 17.3$ \\
\hline Rt. DBP arm, mm Hg & $76.1 \pm 10.4$ & $78.4 \pm 11.0$ & $75.0 \pm 9.9^{*}$ \\
\hline Lt. DBP arm, mm Hg & $75.8 \pm 10.1$ & $78.1 \pm 10.6$ & $74.8 \pm 9.8^{*}$ \\
\hline M. DBP arm, mm Hg & $75.9 \pm 10.1$ & $78.2 \pm 10.6$ & $74.9 \pm 9.7 *$ \\
\hline Rt. SBP lower limb, mm Hg & $147.3 \pm 26.7$ & $152.5 \pm 23.2$ & $144.9 \pm 27.9^{*}$ \\
\hline Lt. SBP lower limb, mm Hg & $145.5 \pm 24.7$ & $151.1 \pm 22.1$ & $143.0 \pm 25.5^{*}$ \\
\hline M. SBP lower limb, mm Hg & $146.4 \pm 24.9$ & $151.8 \pm 22.2$ & $144.0 \pm 25.8^{*}$ \\
\hline Rt. DBP lower limb, mm Hg & $74.0 \pm 10.7$ & $77.0 \pm 10.5$ & $72.6 \pm 10.5^{*}$ \\
\hline Lt. DBP lower limb, mm Hg & $74.7 \pm 10.7$ & $77.1 \pm 10.6$ & $73.6 \pm 10.6^{*}$ \\
\hline M. DBP lower limb, mm Hg & $74.3 \pm 10.4$ & $77.1 \pm 10.4$ & $73.1 \pm 10.2^{*}$ \\
\hline Absolute Dif. SBP arms, mm Hg & $3.2 \pm 3.1$ & $3.0 \pm 2.6$ & $3.3 \pm 3.4$ \\
\hline Relative Dif. SBP arms, mm Hg & $0.7 \pm 4.4$ & $0.8 \pm 3.9$ & $0.6 \pm 4.6$ \\
\hline Absolute Dif. SBP limbs, mm Hg & $7.5 \pm 8.6$ & $6.6 \pm 6.1$ & $7.9 \pm 9.5$ \\
\hline Relative Dif. SBP limbs, mm Hg & $2.3 \pm 11.2$ & $1.6 \pm 8.9$ & $2.4 \pm 12.2$ \\
\hline Absolute Dif. DBP arms, mm Hg & $3.1 \pm 2.7$ & $3.7 \pm 3.0$ & $2.7 \pm 2.6^{*}$ \\
\hline Relative Dif. DBP arms, mm Hg & $0.2 \pm 4.1$ & $0.1 \pm 4.8$ & $0.2 \pm 3.7$ \\
\hline Absolute Dif. DBP limbs, mm Hg & $3.4 \pm 3.3$ & $2.8 \pm 2.6$ & $3.7 \pm 3.5^{*}$ \\
\hline Relative Dif. DBP limbs, mm Hg & $3.2 \pm 3.1$ & $3.0 \pm 2.6$ & $3.3 \pm 3.4$ \\
\hline SBP absolute arms $\geq 10 \mathrm{~mm} \mathrm{Hg,} \mathrm{n}(\%)$ & $12(4)$ & $2(20)$ & $10(5)$ \\
\hline DBP absolute arms $\geq 10 \mathrm{~mm} \mathrm{Hg}, \mathrm{n}(\%)$ & $10(4)$ & $5(16)$ & $5(3)$ \\
\hline SBP absolute lower limbs $\geq 10 \mathrm{~mm} \mathrm{Hg}, \mathrm{n}(\%)$ & $69(24)$ & $17(19)$ & $52(27)$ \\
\hline DBP absolute lower limbs $\geq 10 \mathrm{~mm} \mathrm{Hg}, \mathrm{n}(\%)$ & $1(6)$ & $4(5)$ & $12(6)$ \\
\hline
\end{tabular}

Rt. SBP: right systolic blood pressure; Lt. SBP: left SBP; M. SBP: mean SBP; Rt. DBP: right diastolic BP; Lt. DBP: left DBP; M. DBP: mean DBP; Dif. BP arms: differences in BP between arms; Dif. BP limbs: differences in BP between lower limbs. *P $<0.05$ vs. non-CAD group.

COLIN Co., Ltd, Tokyo, Japan) as described previously [8]. The following equation was used to obtain baPWV: baPWV $=(\mathrm{La}$ (path length from the suprasternal notch to the ankle) $\mathrm{Lb}$ (path length from the suprasternal notch to the brachium))/ $\Delta \mathrm{Tba}$ (time interval between the brachium and ankle). In all studies, baPWV was obtained after at least 5 min of rest. Mean PWV (mPWV) was calculated as (right (rt.) baPWV + left (lt.) baPWV)/2. We measured BP in the arms and lower limbs using a volume-plethysmographic device and calculated the absolute (|rt. BP - lt. BP|) and relative (rt. BP - lt. BP) differences in SBP and DBP between arms.

\section{Statistical analysis}

Statistical analysis was performed using SAS9.3 (SAS Institute Inc.). Data are shown as the mean \pm standard deviation (SD). Categorical and continuous variables were compared between the groups by a Chi-square analysis and unpaired or paired $t$-test, respectively. Multivariate analysis was performed using a logistic regression analysis for independent variables among traditional CV risk factors that were related to the absolute differences in DBP between arms and between lower limbs. A value of $\mathrm{P}<0.05$ was considered significant.

\section{Results}

\section{Patient characteristics in all patients, and in the non-CAD and CAD groups}

Table 1 shows the characteristics in all of the patients, and in the non-CAD and CAD groups. The average age, $\%$ male and BMI in all patients were 66.5 years, $70 \%$ and $23.7 \mathrm{~kg} / \mathrm{m}^{2}$, respectively. There were many significant differences between the non-CAD and CAD groups, including gender, smoking, DL, HTN, DM, and medications including angiotensin II receptor blocker (ARB)/angiotensin converting enzyme inhibitor (ACE-I), calcium channel blocker (CCB), $\beta$-blockers, and 
Table 3. Associations Between baPWV, Absolute Differences in DBP Between Arms, and Other Various Parameters

\begin{tabular}{|c|c|c|c|c|c|c|}
\hline & & (mean) & Absolu & DBP arms & Absc & Dif. DBP limbs \\
\hline & $\mathbf{r}$ & P value & $\mathbf{r}$ & P value & $\mathbf{r}$ & P value \\
\hline Age, years & 0.52 & $<0.0001$ & 0.03 & 0.68 & 0.11 & 0.08 \\
\hline BMI, $\mathrm{kg} / \mathrm{m}^{2}$ & -0.13 & 0.03 & 0.11 & 0.08 & -0.07 & 0.26 \\
\hline M. SBP arm, mm Hg & 0.36 & $<0.0001$ & 0.11 & 0.06 & 0.10 & 0.10 \\
\hline M. DBP arm, mm Hg & 0.12 & 0.04 & 0.09 & 0.15 & 0.02 & 0.72 \\
\hline M. SBP lower limb, mm Hg & 0.42 & $<0.0001$ & 0.04 & 0.46 & -0.02 & 0.78 \\
\hline M. DBP lower limb, mm Hg & 0.27 & $<0.0001$ & 0.05 & 0.44 & -0.12 & 0.04 \\
\hline LDL-C, mg/dL & -0.01 & 0.77 & 0.06 & 0.34 & -0.12 & 0.05 \\
\hline $\mathrm{HDL}-\mathrm{C}, \mathrm{mg} / \mathrm{dL}$ & -0.01 & 0.86 & 0.01 & 0.81 & -0.13 & 0.04 \\
\hline $\mathrm{TG}, \mathrm{mg} / \mathrm{dL}$ & -0.05 & 0.38 & -0.02 & 0.73 & -0.06 & 0.31 \\
\hline HbAlc, $\%$ & 0.14 & 0.02 & -0.11 & 0.06 & 0.01 & 0.83 \\
\hline $\mathrm{eGFR}, \mathrm{mL} / \mathrm{min} / 1.73 \mathrm{~cm}^{2}$ & -0.26 & $<0.0001$ & 0.02 & 0.80 & -0.16 & 0.01 \\
\hline $\mathrm{UA}, \mathrm{mg} / \mathrm{dL}$ & -0.07 & 0.26 & -0.0003 & 0.99 & 0.04 & 0.48 \\
\hline baPWV (mean), cm/s & - & - & -0.04 & 0.50 & 0.05 & 0.41 \\
\hline Absolute Dif. DBP arms, $\mathrm{mm} \mathrm{Hg}$ & -0.04 & 0.50 & - & - & 0.07 & 0.28 \\
\hline Absolute Dif. DBP limbs, mm Hg & 0.05 & 0.41 & 0.07 & 0.28 & - & - \\
\hline
\end{tabular}

BMI: body mass index; M. SBP: mean systolic blood pressure; M. DBP: mean diastolic BP; LDL-C: low-density lipoprotein cholesterol; HDLC: high-density lipoprotein cholesterol; TG: triglyceride; HbA1c: hemoglobin A1c; eGFR: estimated glomerular filtration rate; UA: uric acid; baPWV: brachial-ankle pulse wave velocity; Dif. BP arms: differences in BP between arms; Dif. BP limbs: differences in BP between lower limbs. * $P<0.05$ vs. non-CAD group.

statin. The \% smokers, DL, HTN and DM in the CAD group were significantly higher than those in the non-CAD group. With regard to the use of medications, \% of ARB/ACE-I, $\mathrm{CCB}, \beta$-blockers and statin in the $\mathrm{CAD}$ group were significantly higher than those in the non-CAD group.

\section{Evaluation of baPWV and SBP/DBP in the rt. and lt. arms and lower limbs}

Table 2 shows the baPWV, and SBP/DBP in the rt. and lt. arms and lower limbs in all of the patients, and in the non$\mathrm{CAD}$ and CAD groups. There was no difference in baPWV between the non-CAD and CAD groups. The rt. and 1t. SBP/ DBP in all patients were $130.4 / 76.1$ and $132.8 / 78.4 \mathrm{~mm} \mathrm{Hg}$, respectively. The absolute difference in DBP between arms in the CAD group was significantly lower than that in the nonCAD group, whereas the absolute difference in DBP between lower limbs in the CAD group was significantly higher. There were no differences in the absolute or relative difference in SBP between arms or lower limbs between the CAD and nonCAD groups.

\section{Associations between baPWV, the absolute differences in DBP between arms or lower limbs and various param- eters}

BaPWV was significantly associated with age, BMI, SBP/DBP in the arms and lower limbs, HbA1c and eGFR (Table 3). Although the absolute difference in DBP between lower limbs was significantly associated with HDL-C and eGFR, these associations were very weak. In addition, there was no association between the absolute difference in DBP between arms and between lower limbs.

\section{Associations between the absolute difference in DBP be- tween arms or lower limbs and the Gensini score}

We divided the patients into quartiles according to the Gensini score (Fig. 1). The absolute difference in DBP between arms decreased as the Gensini score increased, as shown in Figure 1A. There was no association between the absolute difference in DBP between lower limbs and the Gensini score (Fig. 1B).

\section{Predictors of the presence of CAD}

To confirm the parameters that predicted the presence of CAD, we performed a logistic regression analysis using independent variables (absolute differences in DBP between arms $<3.1 \mathrm{~mm}$ $\mathrm{Hg}$ (mean values in all patients) and lower limbs $<4.0 \mathrm{~mm} \mathrm{Hg}$ (mean values in all patients) in addition to traditional coronary risk factors (age $\geq 65$ years, gender male, BMI $\geq 25 \mathrm{~kg} / \mathrm{m}^{2}$, DL, DM, HTN, family history and smoking)) that were related to CAD (Fig. 2). CAD was independently associated with the absolute difference in DBP between arms in addition to male, 
A.

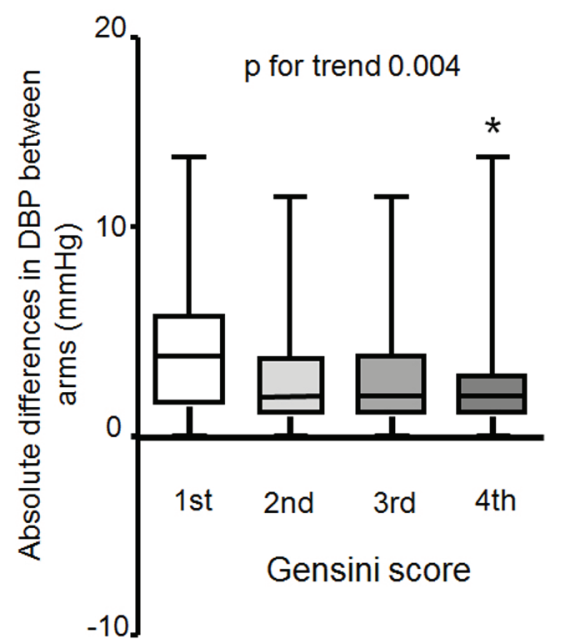

B.

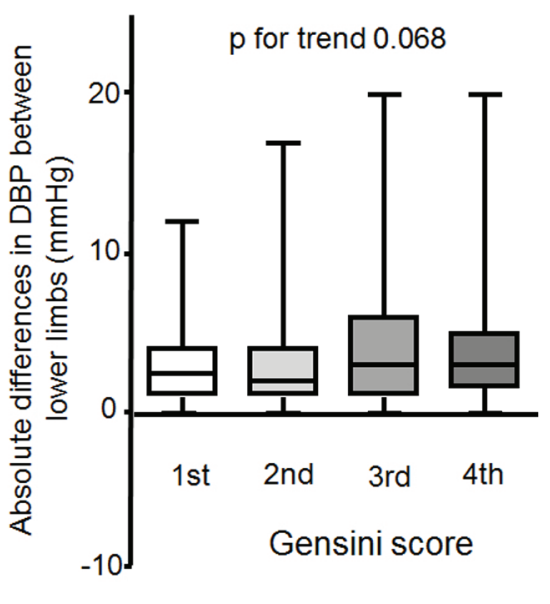

Figure 1. Associations between the absolute difference in DBP between arms (A) or lower limbs (B) and the Gensini score.

family history, DL, DM and HTN.

\section{Discussion}

In this cross-sectional study, we assessed the interrelationship between the absolute and relative differences in BP between arms or lower limbs and the presence of CAD. First, we found that the absolute difference in DBP between arms in patients with $\mathrm{CAD}$ was significantly lower than that in patients without $\mathrm{CAD}$, whereas the absolute difference in DBP between lower limbs in CAD group was significantly higher. Second, the absolute difference in DBP between arms decreased as the Gensini score increased. Finally, among the absolute difference in DBP between arms or lower limbs and traditional CV risk factors (age, male, BMI, smoking, family history, DM, DL and HTN), the absolute difference in DBP between arms, male, family history, HTN, DL and DM were independently associated with the presence of CAD by a logistic regression analysis.

The most interesting finding was that the absolute difference in DBP between arms, but not the absolute difference in SBP between arms, was associated with the severity and presence of CVD. Previous reports have mainly indicated that the difference in SBP, but not DBP, was associated with the severity and presence of CAD and increased CV mortality [3-7]. An absolute difference in BP between arms was associated with $\mathrm{CV}$ risk factors in a general population in Japan [9]. In this study, the absolute difference in SBP between arms was not associated with the severity or presence of CAD. However, traditional CV risk factors (male, family history, HTN, DL and

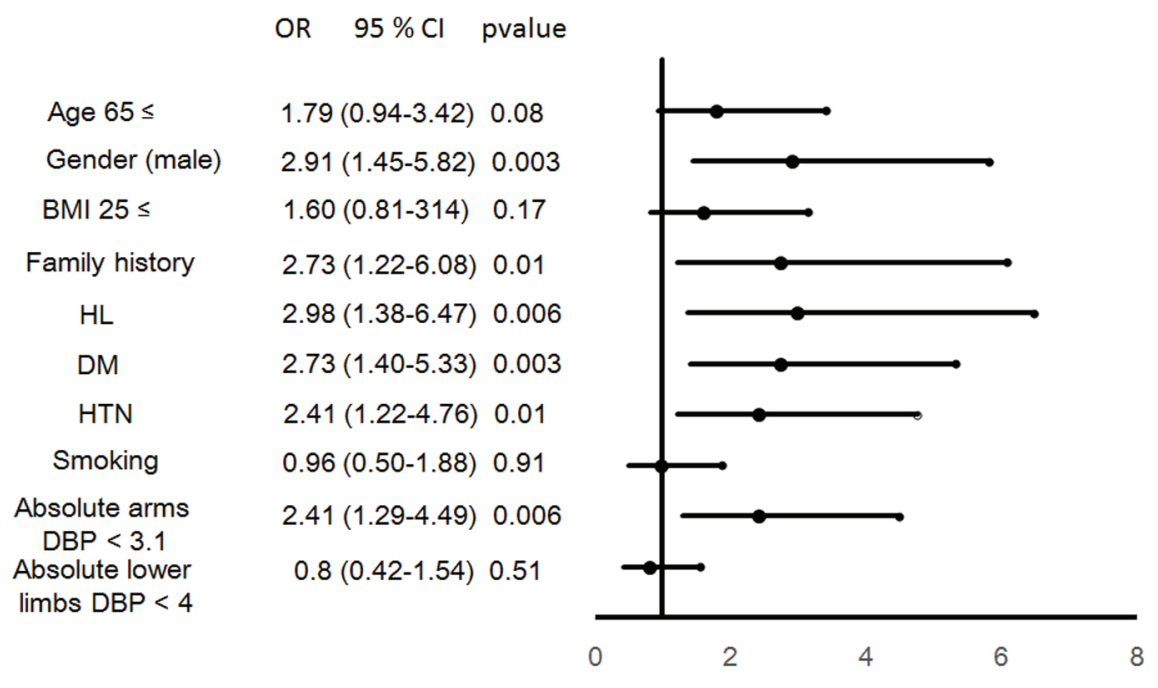

Figure 2. Predictors of the presence of CAD. 
DM) were independent predictors of the presence of CAD. These results indicated that the patients in this study did not represent any specific patient group with CAD. In addition, Clark et al found that $8.6 \%$ of participants with diabetes and $2.9 \%$ of controls had systolic interarm differences $\geq 10 \mathrm{~mm}$ $\mathrm{Hg}$ [10]. Patients with CAD (19.4\%) had a significantly higher prevalence of an absolute BP difference $\geq 10 \mathrm{~mm} \mathrm{Hg}$ than non-CAD patients $(2.7 \%)$ [11]. In this study, the absolute difference in SBP was about $3.2 \mathrm{~mm} \mathrm{Hg}$, and only $4 \%$ of all patients had an absolute SBP difference $\geq 10 \mathrm{~mm} \mathrm{Hg}$. Although we do not know why the absolute difference in SBP was not important for predicting the presence of CAD in this study, a lower prevalence of an absolute BP difference $\geq 10 \mathrm{~mm} \mathrm{Hg}$ may have contributed to this finding. A few reports have stated that the difference in DBP between arms is associated with CV events [3-7]. Among these reports, only Clark et al stated that the difference in DBP between arms was associated with an increased hazard of $\mathrm{CV}$ events and combined non-fatal events or all-cause mortality, albeit with less precision than the difference in SBP between arms. In addition, in this study, a smaller, but not larger, absolute difference in DBP between arms, was associated with the severity and presence of CAD. The population-based Framingham Heart Study revealed that the CAD risk increased with a lower DBP at any level of SBP $\geq 120$ $\mathrm{mm} \mathrm{Hg}$ in the middle-aged and elderly [12]. CV complications may be increased with a fall in BP, especially DBP (J-curve phenomenon between BP and CAD) [13]. Since the coronary arteries are perfused predominantly during diastole, a J-curve, if any, should be most apparent for DBP and CV events. In the CAD group, mean SBP and DBP were 129.0 and $74.9 \mathrm{~mm} \mathrm{Hg}$, respectively (Table 2). Since $\mathrm{DBP}$ in the CAD group was significantly lower than that in the non-CAD group, a lower DBP with a smaller absolute difference in DBP between arms might influence increases in the presence of CAD. Nonetheless, there was no significant association between mean DBP in arms and the absolute difference in DBP between arms in this study ( $r=$ $0.09, \mathrm{P}=0.15$ ) (Table 3). Moreover, it is widely known that $\mathrm{BP}$ is lower in the arm on the side with advanced arteriosclerosis (or atherosclerosis), and such an interarm difference significantly increases as arteriosclerosis advances. This theory also was not consistent with our results. Although this is the first report to show the relationship between a difference in DBP between arms and the severity and presence of CAD, we do not know why a smaller absolute difference in DBP was important for predicting them at this time. Further studies will be needed to clarify this issue.

In a logistic regression analysis, the presence of CAD was independently associated with traditional CV risk factors (male, family history, HTN, DL and DM). Differences in BP between the left and right arms have also been observed in patients with HTN [14] and DM [15]. Although there were no differences in BP between arms between the absence and presence of HTN or DM (data not shown), this may affect the absolute difference in DBP.

\section{Study limitations}

This study has several limitations. First, the study was cross- sectional and included a relatively small number of patients. Second, bilateral BP measurements were performed after various treatments. Many of the patients were taking anti-hypertensive, anti-dyslipidemic and/or anti-diabetic medications that may have influenced the measurements of the differences in BP between arms. It is reasonable to expect that the patients with CAD were taking more medications than non-CAD patients. There were no significant differences in the absolute difference in BP between arms between patients with and without various medications. Prospective studies will be needed to clarify these limitations.

\section{Conclusion}

The absolute difference in DBP between arms in addition to traditional factors may be a critical risk factor for the presence of CAD.

\section{Disclosure}

K.S. is a Chief Director and S.M. is a Director of NPO Clinical and Applied Science, Fukuoka, Japan. K.S. has an Endowed Department of "Department of Molecular Cardiovascular Therapeutics" supported by MSD, Co. Ltd. S.M. belongs to the Department of Molecular Cardiovascular Therapeutics supported by MSD, Co. Ltd.

\section{References}

1. Shimamoto K, Ando K, Fujita T, Hasebe N, Higaki J, Horiuchi $\mathrm{M}$, Imai Y, et al. The Japanese Society of Hypertension Guidelines for the Management of Hypertension (JSH 2014). Hypertens Res. 2014;37(4):253-390.

2. Eguchi K, Yacoub M, Jhalani J, Gerin W, Schwartz JE, Pickering TG. Consistency of blood pressure differences between the left and right arms. Arch Intern Med. 2007;167(4):388-393.

3. Agarwal R, Bunaye Z, Bekele DM. Prognostic significance of between-arm blood pressure differences. Hypertension. 2008;51(3):657-662.

4. Clark CE, Campbell JL, Evans PH, Millward A. Prevalence and clinical implications of the inter-arm blood pressure difference: A systematic review. J Hum Hypertens. 2006;20(12):923-931.

5. Clark CE, Powell RJ. The differential blood pressure sign in general practice: prevalence and prognostic value. Fam Pract. 2002;19(5):439-441.

6. Clark CE, Taylor RS, Shore AC, Campbell JL. The difference in blood pressure readings between arms and survival: primary care cohort study. BMJ. 2012;344:e1327.

7. Yamamoto T, Miura SI, Suematsu Y, Kuwano T, Sugihara M, Ike A, Iwata A, et al. A relative difference in systolic blood pressure between arms by synchronal measurement and conventional cardiovascular risk factors are associated with the severity of coronary atherosclerosis. Heart Vessels. 2015. 
8. Mitsutake R, Miura S, Saku K. Association between coronary artery calcification score as assessed by multi-detector row computed tomography and upstroke time of pulse wave. Intern Med. 2007;46(22):1833-1836.

9. Kimura A, Hashimoto J, Watabe D, Takahashi H, Ohkubo T, Kikuya M, Imai Y. Patient characteristics and factors associated with inter-arm difference of blood pressure measurements in a general population in Ohasama, Japan. J Hypertens. 2004;22(12):2277-2283.

10. Clark CE, Steele AM, Taylor RS, Shore AC, Ukoumunne OC, Campbell JL. Interarm blood pressure difference in people with diabetes: measurement and vascular and mortality implications: a cohort study. Diabetes Care. 2014;37(6):1613-1620.

11. Orme S, Ralph SG, Birchall A, Lawson-Matthew P, McLean K, Channer KS. The normal range for inter-arm differences in blood pressure. Age Ageing. 1999;28(6):537-542.
12. Franklin SS, Khan SA, Wong ND, Larson MG, Levy D. Is pulse pressure useful in predicting risk for coronary heart Disease? The Framingham heart study. Circulation. 1999;100(4):354-360.

13. Messerli FH, Panjrath GS. The J-curve between blood pressure and coronary artery disease or essential hypertension: exactly how essential? J Am Coll Cardiol. 2009;54(20):1827-1834.

14. Clark CE, Campbell JL, Powell RJ. The interarm blood pressure difference as predictor of cardiovascular events in patients with hypertension in primary care: cohort study. J Hum Hypertens. 2007;21(8):633-638.

15. Kleefstra N, Houweling ST, Meyboom-de Jong B, Bilo HJ. [Measuring the blood pressure in both arms is of little use; longitudinal study into blood pressure differences between both arms and its reproducibility in patients with diabetes mellitus type 2]. Ned Tijdschr Geneeskd. 2007;151(27):1509-1514. 